\title{
HIGHLIGHTS
}

CANCER

\section{MicroRNA expression provides clues about the aggressiveness of papillary thyroid carcinoma}

The expression level of certain microRNAs (miRNAs) in papillary thyroid carcinoma (PTC) tissue samples indicate some of the tumor's clinicopathogenetic features, demonstrate Chou and co-investigators in a recent article published in Thyroid.

Dysregulation of miRNAs is involved in the pathogenesis of human cancers including breast, lung and PTC. Specifically in PTC, miR-221, miR-222 and miR-146b are overexpressed compared to the level in normal thyroid tissue; however, the target genes regulated by these miRNA have not been fully elucidated and associations between clinicopathogenetic features of PTC and expression of specific miRNAs are unclear.

The Taiwanese researchers analyzed the expression levels of miRNAs miR-221, miR-222 and miR-146b in tumor tissue samples from 100 patients with PTC with distinct clinicopathogenetic properties and in normal thyroid tissue samples from 16 paired individuals without PTC.
Patients were designated as having highrisk ( $\geq 45$ years old with stage I or II PTC) or low-risk ( $<45$ years old with stage I PTC) cancer.

(4) ...alterations in miRNA expression may provide ... a selective growth advantage... 77

The findings revealed associations between miRNA expression levels and various high-risk features of PTCs. Firstly, expression levels of all three miRNAs were significantly higher in PTCs with extrathyroidal invasion than those without invasion. Secondly, expression levels of two of the miRNAs (miR-221 and miR-146b) were higher in the high-risk group than the low-risk group. Lastly, PTCs with BRAF mutations - an indicator of high aggressiveness-had increased expression of miR-146b.

"The results support the hypothesis that alterations in miRNA expression may provide cancer with a selective growth advantage to develop aggressive properties," points out senior researcher Rue-Tsuan Liu of Chang Gung University, Taiwan. "The same phenomenon has been reported in primary breast carcinomas."

The researchers now plan to identify miR-146b-regulated target genes associated with tumor aggressiveness. Their current and future findings promise advances in the prognosis and treatment of PTC. "Not only may the miR-146b expression profile be adopted as a novel prognostic biomarker for patients with PTCs, it may also serve as a therapeutic target and enable us to identify possible miR-146b-regulated target gene(s) associated with tumor aggressiveness," concludes Liu.

\section{Carol Wilson}

Original article Chou, C. K. et al. miR-146b is highly expressed in adult papillary thyroid carcinomas with high risk features including extrathyroidal invasion and the BRAF(V600E) mutation. Thyroid 20, 489-494 (2010) 